\title{
Load and Velocity Dependence of Friction Mediated by Dynamics of Interfacial Contacts
}

\author{
Wengen Ouyang, ${ }^{1}$ Shivaprakash N. Ramakrishna, ${ }^{2 *}$ Antonella Rossi,${ }^{2,3}$ Michael Urbakh, ${ }^{1}$ \\ Nicholas D. Spencer, ${ }^{2}$ and Andrea Arcifa $\oplus^{2 \dagger, \$}$ \\ ${ }^{1}$ School of Chemistry and The Sackler Center for Computational Molecular and Materials Science, \\ Tel Aviv University, Tel Aviv 69978, Israel \\ ${ }^{2}$ Laboratory for Surface Science and Technology, Department of Materials, ETH Zurich, \\ Vladimir-Prelog-Weg 5,CH-8093 Zurich, Switzerland \\ ${ }^{3}$ Dipartimento di Scienze Chimiche e Geologiche, Università degli Studi di Cagliari, \\ Cittadella Universitaria di Monserrato, I-09100 Cagliari, Italy
}

(Received 17 March 2019; revised manuscript received 19 June 2019; published 9 September 2019)

\begin{abstract}
Studying the frictional properties of interfaces with dynamic chemical bonds advances understanding of the mechanism underlying rate and state laws, and offers new pathways for the rational control of frictional response. In this work, we revisit the load dependence of interfacial chemical-bond-induced (ICBI) friction experimentally and find that the velocity dependence of friction can be reversed by changing the normal load. We propose a theoretical model, whose analytical solution allows us to interpret the experimental data on timescales and length scales that are relevant to experimental conditions. Our work provides a promising avenue for exploring the dynamics of ICBI friction.
\end{abstract}

DOI: 10.1103/PhysRevLett.123.116102

Friction plays a central role in a broad variety of systems, whose characteristic length scales and timescales vary from those of molecular motors [1,2] and nano- or micromechanical devices $[1,3]$, to those relevant to transportation, industrial machines [4], and geophysical phenomena [5]. The search for technological solutions to control friction, and the related phenomenon of wear, has been pivotal to the progress of humanity $[6,7]$.

In recent decades, the development of highly sensitive experimental tools, such as the atomic force microscope (AFM) and the surface forces apparatus (SFA), has played a fundamental role in advancing our understanding of friction [8]. In particular, AFM-based approaches provide the opportunity to probe the tribological behavior of singleasperity-like contacts under diverse environmental and operating conditions [3,9-15]. Such measurements have often revealed unexpected frictional behavior that goes beyond the predictions of the Prandtl-Tomlinson (PT) model [14,16-20], which for decades has been the most influential concept in the field of nanotribology $[10,21,22]$. These observations have stimulated a reconsideration of the basic assumptions of the PT paradigm and led to the development of novel, coarse-grained models, which have yielded remarkable insights into nanoscale friction $[16,23-25]$, as well as wear $[14,26,27]$ and tribofilm formation [15].

In the present work, we focus on understanding the mechanisms of an unexpected frictional phenomenon observed in our lateral force microcopy (LFM) investigation of a silicon tip sliding against a silicon surface immersed in an ionic liquid (IL). The experimental results showed a strong nonlinear variation of friction with normal load (non-Amontons' behavior) and a dramatic change in the friction with respect to sliding speed for different normal loads. We interpret our result based on the "multicontact" model (MCM) proposed by Filippov et al. [28] and Barel et al. [16]. We extend the MCM and derive an analytical equation for the frictional force as a function of both sliding speed and normal load, which agrees well with the results of experiments.

Our experiments involved the frictional behavior of a sharp, silicon AFM tip immersed in 1-ethyl-3 methylimidazolium bis(trifluoromethylsulfonyl)imide ([EMIM] TFSI), sliding against the (100) face of a $\mathrm{O}_{2}$-plasma-treated silicon wafer (details of experimental setup and sample characterization can be found in Sec. 1 of the Supplemental Material [29]). All friction experiments presented here were carried out at room temperature $(298 \pm 2 \mathrm{~K})$, under a constant flow of nitrogen. This purging procedure allowed us to reach a relative humidity $(\mathrm{RH})$ value of $4 \pm 2 \%$. As reported earlier $[45,46]$ a substantial decrease of adhesion was observed, in comparison with the values measured in air (see Figs. S3 and S4 in the Supplemental Material [29]).

Our previous friction-vs-load experiments [46], carried out under conditions analogous to those discussed in the present work, pointed to a substantial increase in the coefficient of friction $\mu$-defined here as the gradient of the friction-vs-load curve-at an applied load of a few $\mathrm{nN}$, which was tentatively interpreted as an indication of the stress-promoted formation and rupture of covalent bonds at the sliding interface. In the present study, we delve further into these observations and hypotheses by presenting 


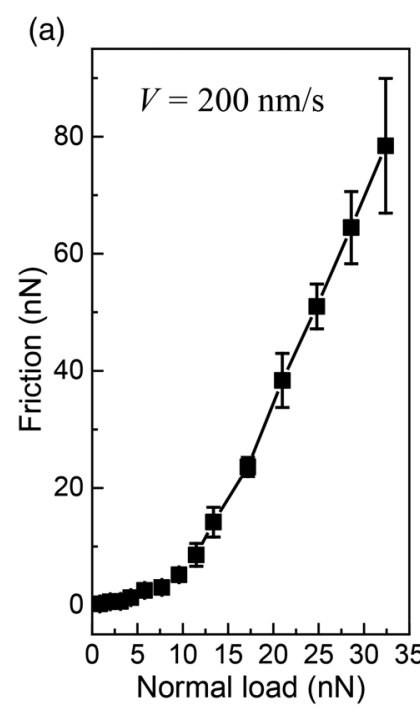

(b)

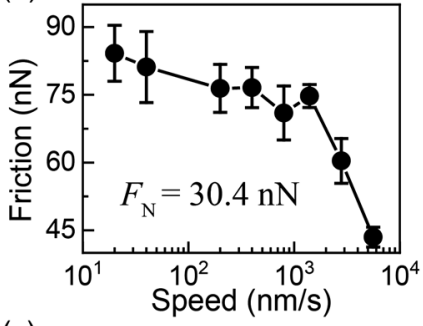

(c)

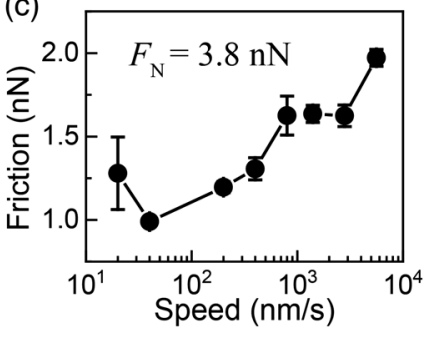

FIG. 1. Dependence of friction on normal load and speed for a silicon AFM tip sliding on the surface of a silicon (100) wafer under [EMIM] TFSI. (a) The friction force as a function of normal load, which was obtained by progressively decreasing the load (35 to $0 \mathrm{nN}$ ), at a constant speed of $200 \mathrm{~nm} / \mathrm{s}$. (b) and (c) The velocity dependencies of friction force measured under normal load at 30.4 and $3.8 \mathrm{nN}$, respectively. All experimental data shown in the figure were acquired with the same cantilever (normal spring constant of $0.082 \mathrm{~N} / \mathrm{m}$ ) and silicon wafer, under a continuous nitrogen flow $(T=298 \pm 2 \mathrm{~K}, \mathrm{RH}=4 \pm 2 \%)$. The lines are plotted to guide the eye.

additional experimental results, and use the extended MCM to obtain a more detailed description of the frictional behavior of a silicon-based nanoscale contact sliding in the presence of a hydrophobic IL.

Figure 1 shows the dependence of friction on normal load [Fig. 1(a)] and speed [Figs. 1(b)-(c)] measured by LFM. Details of the LFM experiments are reported in the Supplemental Material [29].

Figure 1(a) shows a strong nonlinear dependence of friction with the normal load. The pronounced convexity of the trend observed at low normal loads cannot be explained by the assumption of the friction-contact area proportionality, as projected by continuum-contact-mechanics models [3,47-50]. Figures 1(b)-1(c) show the speed dependence of friction under two different loads. Under high normal load $(30.4 \mathrm{nN})$, the friction decreases with increasing velocity, while under low normal load $(3.8 \mathrm{nN})$, the friction increases with increasing velocity. A decrease in friction with velocity has been also observed in previous AFM experiments [16,51], and was correlated to the rupture and reformation of molecular contacts, which can be reproduced by stochastic simulations based on the MCM. We believe that the friction-load and friction-velocity behavior observed in our experiments also originate from the rupture and reformation of nanoscale contacts, and we propose an extended MCM that includes the influence of applied load on friction. Here, we consider the MCM of the kind

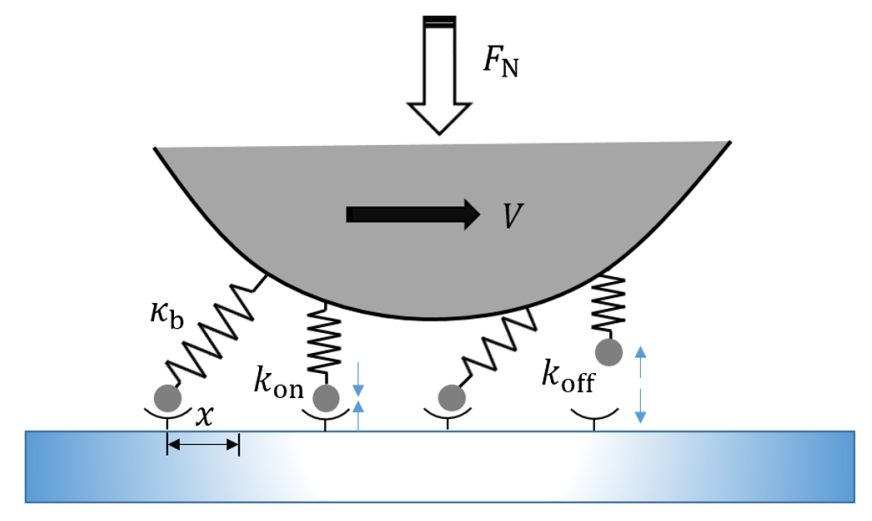

FIG. 2. Schematic of the multicontact model. $k_{\text {on }}$ and $k_{\text {off }}$ are the rates for binding and unbinding, respectively. $\kappa_{b}$ and $x$ are the stiffness and elongation of a single bond, respectively. $F_{N}$ is the applied normal load.

depicted in the schematic representation, Fig. 2, which describes the interaction between an AFM tip and the underlying surface through an array of contacts representing molecular bonds, when a constant normal load $F_{N}$ is applied to the tip.

As shown in previous studies, it is assumed that the rupture and reattachment of contacts are thermally activated processes $[16,25,28,52-55]$. Additionally, when one of the surfaces is laterally pulled over the other, the tensile force acting on the interfacial contacts reduces the activation energy necessary for contact rupture [16,28,54,56,57]. The corresponding analytical expressions for the rates of formation $\left(k_{\mathrm{on}}\right)$ and rupture $\left(k_{\mathrm{off}}\right)$ of contacts are the following:

$$
\begin{aligned}
& k_{\text {on }}=k_{\text {on }}^{0} e^{-\left[\left(\Delta E_{\mathrm{on}}\right) /\left(k_{B} T\right)\right]}, \\
& k_{\text {off }}=k_{\text {off }}^{0} e^{-\left[\left(\Delta E_{\text {off }}-\kappa_{b} x_{s} x\right) /\left(k_{B} T\right)\right]},
\end{aligned}
$$

where $k_{\mathrm{on}}^{0}$ and $\Delta E_{\mathrm{on}}$ are the attempt frequency and the barrier height for the reattachment, $k_{\text {off }}^{0}$ and $\Delta E_{\text {off }}$ are the characteristic attempt frequency and the height of potential barrier for breaking a contact in the absence of the external force, $\kappa_{b}$ and $x$ are the stiffness and elongation of an intact bond, $x_{s}$ is the minimum-to-barrier distance in the contactsurface interaction [56,57], $k_{B}$ is the Boltzmann constant, and $T$ the absolute temperature.

The resistance force experienced by the tip ("steady-state friction"), resulting from the elastic stretching of intact (unbroken) bonds in the lateral direction can be expressed analytically $[58,59]$ as

$$
F^{\text {single }}(B)=N \frac{k_{B} T}{x_{s}} \frac{r_{\text {on }} B e^{B} Q(B)}{1+r_{\text {on }} B e^{B} E_{1}(B)},
$$

where the superscript "single" is used to emphasize that all the contacts belong to the same type-i.e., they are characterized by the same set of kinetic parameters, $N$ is the total number of contacts, and $r_{\text {on }}=\left(k_{\text {on }}^{0} / k_{\text {off }}^{0}\right) \exp \left\{\left[\left(\Delta E_{\text {off }}-\right.\right.\right.$ $\left.\left.\left.\Delta E_{\text {on }}\right) / k_{B} T\right]\right\}$ is the ratio between $k_{\text {on }}$ and $k_{\text {off }} . E_{1}(B)=$ $\int_{B}^{\infty} t^{-1} e^{-t} d t$ is the exponential integral and 
$Q(B)=\int_{1}^{\infty} t^{-1} e^{-B t} \ln t d t$, where $B=\left(k_{B} T / \kappa_{b} x_{s} V\right) k_{\mathrm{off}}^{0} \times$ $\exp \left(-\Delta E_{\text {off }} / k_{B} T\right)$. The details of the derivation of Eq. (2) can be found in Sec. 5.1 of Supplemental Material [29].

In Eq. (2) we include temperature effect explicitly-this equation agrees well with the results of direct stochastic simulations over a broad range of pulling speeds and temperatures [16] — and has proven to be useful to gain insights concerning the dependency of frictional response on temperature and sliding speed [16,23,60-62]. In the following, we extend the model to consider the effect of normal load on friction.

We first notice that increasing normal load leads to an enlargement of the contact area by elastic deformation, which translates into an increase of the number of reaction sites available for contact formation. Second, we consider the possible role of contact pressure on the kinetics of interfacial-bond formation. In this respect, several authors have recently proposed that the effect of applied load on single-asperity tribological phenomena, such as frictional aging and atomic attrition [14,20,26,63], can be rationalized by considering the dependency of activation energy of the bond formation process on the contact pressure of the type $\Delta E_{\mathrm{on}}=\Delta E_{\mathrm{on}}^{0}-p V_{a}$, where $\Delta E_{\mathrm{on}}^{0}$ is the activation energy for bond formation at zero pressure, $p$ is the contact pressure, and $V_{a}$ is a parameter that is usually interpreted as the activation volume $[14,20,26]$.
We include both these effects in our extended MCM, and further assume that the deformation of the asperity against the flat can be described by the Hertzian contact theory [64]. Accordingly, the radius of the contact area and the distribution of the contact pressure is expressed as $a=$ $\left(3 R_{\text {tip }} F_{N} / 4 E^{*}\right)^{1 / 3}$ and $p\left(r ; F_{N}\right)=p_{0}\left(F_{N}\right)\left(1-r^{2} / a^{2}\right)^{1 / 2}$, respectively. Here, $p_{0}\left(F_{N}\right)=3 F_{N} / 2 \pi a^{2}$ is the normal stress at the center of the contact area, $E^{*}$ is the effective Young's modulus of the contact: $1 / E^{*}=\left(1-\nu_{1}^{2}\right) / E_{1}+$ $\left(1-\nu_{2}^{2}\right) / E_{2}$, and $\nu_{1}$ and $\nu_{2}$ are the Poisson's ratios of the tip and the surface, respectively. A discussion of the limitations of our model can be found in Sec. 5.3 of the Supplemental Material [29].

For a uniform distribution of binding sites within the contact area, their radial density distribution can be written as $\rho(r)=n_{0} 2 \pi r, 0 \leq r \leq a$. Under the above conditions and Eq. (2), the friction force accounting for a distribution of activation energies $\Delta E_{\text {on }}$ can be calculated as

$$
\bar{F}_{\mathrm{ss}}=n_{0} 2 \pi \int_{0}^{a} r \frac{k_{B} T}{x_{s}} \frac{r_{\mathrm{on}} B e^{B} Q(B)}{1+r_{\mathrm{on}} B e^{B} E_{1}(B)} d r
$$

Then, after integration, we arrive at the following equation for the steady-state friction force:

$$
\bar{F}_{\text {ss }}\left(F_{N}, V ; n_{0}, V_{a}, \cdots\right)=\frac{2 n_{0} \pi a^{2}}{x_{0}^{2}} \frac{k_{B} T}{x_{s}} \frac{Q(B)}{E_{1}(B)}\left[x_{0} \ln \left(1+D e^{x_{0}}\right)-\mathrm{Li}_{2}(-D)+\mathrm{Li}_{2}\left(-D e^{x_{0}}\right)\right],
$$

where, $\quad x_{0}=p_{0}\left(F_{\mathrm{N}}\right) V_{a} / k_{B} T$ and $\quad D=r_{\text {on }}(p=$ 0) $B e^{B} E_{1}(B)$ and $\mathrm{Li}_{2}(z)=-\int_{0}^{z} t^{-1} \ln (1-t) d t$ is the dilogarithm [65].

The asymptotic expansion of Eq. (4) shows that, at sufficiently high loads, friction force scales as $\bar{F}_{\text {ss }} \sim F_{N}^{2 / 3}$; i.e., it shows the same dependency on load as that predicted by Hertzian theory for the contact area. At sufficiently low normal loads, the model predicts a dependency of the type $\bar{F}_{\text {ss }} \sim F_{N}^{1 / 3}$, which differs significantly from the predictions based on Hertz contact theory.

Figure 3 illustrates the normal load and speed dependency of friction predicted by Eq. (4) for parameters listed in the caption of Fig. 4 (see also Table SI in the Supplemental Material [29]) for the primary contacts. To better portray asymptotic behaviors of friction, numerical calculations are reported over a broad range of normal loads and speeds. In reality, extreme values of speed or load may be unattainable in LFM experiments, or they may lead to the occurrence of additional mechanisms that cannot be considered by the model, such as frictional heating and plastic deformation.

Figure 3 demonstrates that the extended MCM captures the nonlinear dependence of friction on the normal load found in our experiments, but fails to describe the observed dramatic effect of normal load on the speed dependence of friction. Specifically, the MCM predicts similar speed dependencies of friction in both the low- and high-load regimes [see Fig. 3(b)], which contrasts with our observations.

On the other hand, it should be noted that the simplified model considered in Fig. 2 accounts for a single type of interaction between sliding counterparts. In reality, multiple dissipative mechanisms may contribute to the overall
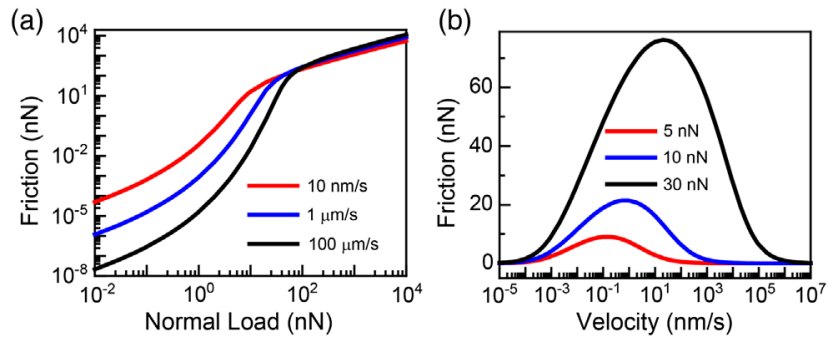

FIG. 3. Friction force as a function of normal load for different sliding speeds (a), and of sliding speed for different normal loads (b). The curves are predicted by Eq. (4) using the parameters listed in the caption of Fig. 4 for the primary contacts. 

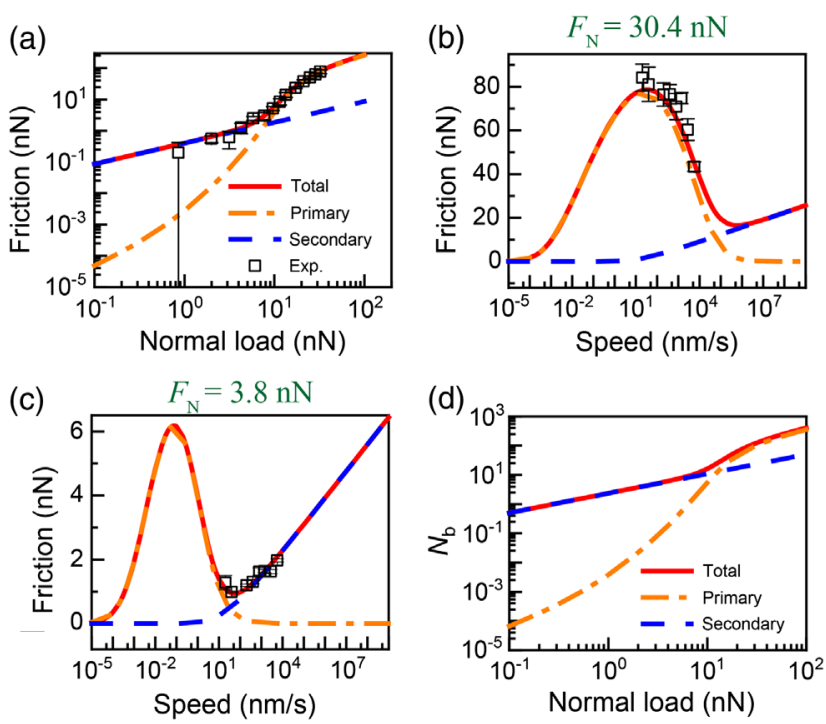

FIG. 4. Comparison of theoretical results and the experimental data from in Fig. 1. Dependence of friction on normal load (log$\log$ ) (a) and speed (semilog) (b)-(c) for a silicon AFM tip and the surface of a silicon (100) wafer under [EMIM] TFSI. Panel (d) illustrates the number of intact contacts $\left[N_{b}\right.$, see Eq. (S9) in the Supplemental Material [29]] as a function of normal load. Experimental data are represented by black squares. The red solid line is the fitting curve obtained from Eq. (5). The orange dashdotted line and blue dashed line represent the contribution to friction force of primary and secondary contacts, respectively. Fitting parameters for primary contacts: $E_{\text {on }}^{\mathrm{I}}=0.87 \mathrm{eV}, E_{\text {off }}^{\mathrm{I}}=$ $0.78 \mathrm{eV}, \kappa_{b}^{\mathrm{I}}=10 \mathrm{~N} / \mathrm{m}, n_{0}^{\mathrm{I}}=7.92 \mathrm{~nm}^{-2}$, and $V_{a}^{\mathrm{I}}=43.2 \AA^{3}$. Fitting parameters for secondary contacts are $E_{\mathrm{on}}^{\mathrm{II}}=0.04 \mathrm{eV}$, $E_{\text {off }}^{\mathrm{II}}=0.58 \mathrm{eV}, \kappa_{b}^{\mathrm{II}}=1 \mathrm{~N} / \mathrm{m}, n_{0}^{\mathrm{II}}=1 \mathrm{~nm}^{-2}$, and $V_{a}^{\mathrm{II}}=24.5 \AA^{3}$. Other parameters are identical for both types of contacts: $k_{\mathrm{on}}^{0}=k_{\mathrm{off}}^{0}=10^{13} \mathrm{~Hz}, x_{s}=0.2 \mathrm{~nm}, R_{\mathrm{tip}}=40 \mathrm{~nm}, E^{*}=35.3 \mathrm{GPa}$, and $T=298 \mathrm{~K}$.

friction. The dramatic change of the friction-velocity behavior in low- and high-load regimes observed in our experiments may suggests that two types of mechanism contribute to friction. Within the framework of the MCM, each one can be modeled by a distinct set of contacts, type I and II in the following. Assuming that these two contributions have independent binding sites, the corresponding MCM can be expressed as

$$
\begin{aligned}
\bar{F}_{\mathrm{ss}}^{\mathrm{tot}}= & \bar{F}_{\mathrm{ss}}\left(F_{N}, V ; n_{0}^{\mathrm{I}}, V_{a}^{\mathrm{I}}, E_{\mathrm{on}}^{\mathrm{I}}, E_{\mathrm{off}}^{\mathrm{I}}, \kappa_{b}^{\mathrm{I}}\right) \\
& +\bar{F}_{\mathrm{ss}}\left(F_{N}, V ; n_{0}^{\mathrm{II}}, V_{a}^{\mathrm{I}}, E_{\mathrm{on}}^{\mathrm{II}}, E_{\mathrm{off}}^{\mathrm{II}}, \kappa_{b}^{\mathrm{II}}\right) .
\end{aligned}
$$

In Eq. (5) the functions $\bar{F}_{\mathrm{ss}}\left(F_{N}, V ; n_{0}^{\mathrm{I}}, V_{a}^{\mathrm{I}}, E_{\mathrm{on}}^{\mathrm{I}}, E_{\text {off }}^{\mathrm{I}}, \kappa_{b}^{\mathrm{I}}\right)$ and $\bar{F}_{\text {ss }}\left(F_{N}, V ; n_{0}^{\mathrm{II}}, V_{a}^{\mathrm{I}}, E_{\mathrm{on}}^{\mathrm{II}}, E_{\mathrm{off}}^{\mathrm{II}}, \kappa_{b}^{\mathrm{II}}\right)$ are both described by Eq. (4); the apex I and II refer to the two types of contact, each one characterized by a set of parameters, which are described above.

Figure 4 displays the results of fitting the experimental data using Eq. (5), with the simulation parameters reported in its caption and Table SI in the Supplemental Material [29]. A discussion of the physically motivated constraints on the fitting parameters and the details of the fitting procedure can be found in Sec. 6 of the Supplemental Material [29].

The "primary" (type I) contribution, represented by the orange dash-dotted line in Fig. 4, captures the experimental behavior observed for sufficiently high applied loads [>10 nN $(\sim 1.1 \mathrm{GPa})$, see Fig. 4(a)] and low speeds $\left[<10^{3} \mathrm{~nm} / \mathrm{s}\right.$, Fig. 4(b)]. Interestingly, the energy barrier for bond formation obtained by fitting the experimental data is $0.87 \mathrm{eV}$ (see the Supplemental Material [29]), which is located within the range expected for forming a siloxane bond (Si-O-Si), as estimated from kinetic Monte Carlo simulations $(0.68-1.3 \mathrm{eV})$ [20]. In addition, the estimated energy barrier for breaking primary contacts obtained with our model is $0.78 \mathrm{eV}$, which is also in the range of 0.32 $1.27 \mathrm{eV}$ obtained by a number of classical MD and ab initio simulation methods [66] for breaking a siloxane bond. This comparison suggests that, under sufficiently high applied loads and low speeds, the measured friction is mainly due to the formation of interfacial covalent bonds, possibly siloxane bridges. Our simulations show [see Fig. 4(d)] that the number of strong contacts in the bound state is negligible for loads below $10 \mathrm{nN}(\sim 1.1 \mathrm{GPa})$, indicating that the primary contacts do not form in the low-load regime.

The introduction of a "secondary" (type II) contact (blue dashed line) significantly improves the agreement between the model and the experimental results over the entire range of loads and speeds investigated in this study. Notably, in order to reproduce the trend observed in Fig. 4(c), the formation of the secondary contacts is expected to be a weakly activated process $\left(\Delta E_{\mathrm{on}}^{\mathrm{II}} \sim k_{B} T\right)$, such that the rate of contact formation approaches the attempt frequency $k_{\mathrm{on}}^{0}$, and the speed dependence of friction is determined by the detachment of the weak contacts. The above statement can be proven analytically: for $B \ll 1$ and $D \gg 1$ [see Eq. (4)] the asymptotic expansion of Eq. (4) shows that the friction force scales as $F \sim Q(B) / E_{1}(B) F_{\mathrm{N}}^{2 / 3} \sim F_{\mathrm{N}}^{2 / 3} \ln V$. The logarithmic dependency of friction on speed found under these conditions is comparable to the trend commonly predicted by PT-like models [22]. By comparing Figs. 3(b) and 4(d), we found a qualitative difference between the speed dependence of friction for a single type of contact [Eq. (4)] and two types of contact [Eq. (5)]. For a single type of contact, the friction force first increases and then decreases with increasing velocity, while for two types of contact, the behavior is more complicated, the friction force first increases and then decreases with increasing velocity, as the velocity grows it increases again. This qualitative difference might be exploited for identifying whether the type of contact is single or not.

In our experiments, both the silicon surface and the silicon AFM tip are hydroxylated (see details of surface 
characterization in Sec. 1 of the Supplemental Material [29]), so we expect the formation of siloxane bonds between the silanol groups under conditions leading to direct contact between counterparts. As discussed above, the similarity between the values of the fitting parameters used for modeling rupture and reattachment of primary contacts (see the Supplemental Material [29]), with those reported in references for the description of frictional aging of nanoscale silica surfaces $[19,20,66]$, point to a similar microscopic origin for these phenomena, substantiating our hypothesis.

Although the contribution due to rupture and reattachment of secondary contacts is most probably related to noncovalent, weakly activated interactions, gaining detailed understanding of the molecular nature of those interactions is challenging.

Intermolecular forces (i.e., van der Waals and hydrogenbond interactions) between opposing silica surfaces might be expected if the AFM tip penetrates the liquid layer over the entire range of normal loads accessible in this work. On the other hand, SFA-based experiments have demonstrated that a film of ILs confined between mica counterparts resists squeezing out up to at least $\sim 1 \mathrm{MPa}$ of contact pressure [67-70]. The presence of a confined-boundary - film of ionic liquid has been assumed in a number of LFM-based investigations on IL-mediated lubrication [7173], although, typically, the AFM technique cannot provide unambiguous information concerning the absolute separation (i.e., the presence of a boundary layer) between solid counterparts.

With the above discussions, we can propose a physical picture for the experimental observations reported in this work. For sufficiently low normal loads $(<10 \mathrm{nN})$, sliding is likely to occur between adsorbed ions located between the tip and the surface [see Fig. 4(d)], leading to a PT-like dependency of friction on speed, and a slow increase of friction with normal load. When the normal loads become sufficiently high $(>10 \mathrm{nN})$, the IL between the counterparts is then squeezed out, exposing silanol groups on the surfaces. As a result of stress-promoted, thermally activated rupture and reattachment of interfacial covalent (siloxane) bonds, the friction force is expected to decrease with sliding velocity over the investigated range of loads, and to grow with normal load much faster than in the case of the lowload regime. Although the MCM assumes that the two types of contacts can form independently, the contributions of the primary and secondary contacts to the friction force are negligible in the low-load and high-load regimes, respectively [see Figs. 4(b)-4(c)]. Under these conditions, the predictions of the model are essentially equivalent to those expected for the physical picture described above.

In conclusion, we have shown that the frictional properties of $\mathrm{SiO}_{2}$-based contacts lubricated with a hydrophobic IL can be properly described by an extended MCM that includes two distinct types of interfacial contact.
The outcomes of the model suggest that the stress-assisted, thermally activated formation and rupture of interfacial contacts dominate the frictional behavior at sufficiently high contact pressures; the contribution of weaker and nonactivated interactions prevails for low loads and high speeds, as the contribution due to the covalent bonds becomes negligible.

The analytical solution provides a framework for the interpretation of the experimental data on the time and length scales relevant to tribological experimental conditions, which is generally impractical when using numerical simulations. Our work provides a promising avenue for further exploration of the normal load and velocity dependence of friction at interfaces involving dynamic bond formation and rupture.

W. O. acknowledges financial support from the Planning and Budgeting Committee fellowship program for outstanding postdoctoral researchers from China and India in Israeli Universities. M. U. acknowledges financial support of the Israel Science Foundation, Grant No. 1141/18, and of the Deutsche Forschungsgemeinschaft (DFG), Grant No. BA 1008/21-1. This work is supported in part by COST Action MP1303. The authors thank Professor Dr. Roland Bennewitz and Professor Dr. Rosa M. Espinosa-Marzal for their constructive comments.

*Corresponding author.

shivaprakash.ramakrishna@mat.ethz.ch

Corresponding author.

andrea.arcifa@empa.ch

${ }^{\star}$ Present address: EMPA, Swiss Federal Institute for Material Science and Technology, Switzerland.

[1] M. Urbakh, J. Klafter, D. Gourdon, and J. Israelachvili, Nature (London) 430, 525 (2004).

[2] V. Bormuth, V. Varga, J. Howard, and E. Schäffer, Science 325, 870 (2009).

[3] Y. Mo, K. T. Turner, and I. Szlufarska, Nature (London) 457, 1116 (2009).

[4] K. Holmberg, P. Andersson, and A. Erdemir, Tribol. Int. 47, 221 (2012).

[5] C. H. Scholz, Nature (London) 391, 37 (1998).

[6] B. Wilson, Ind. Lubr. Tribol. 50 (1998).

[7] D. Dowson, History of Tribology (Addison-Wesley Longman Limited, Reading, MA, 1979).

[8] M. Urbakh and E. Meyer, Nat. Mater. 9, 8 (2010).

[9] E. Gnecco, R. Bennewitz, T. Gyalog, C. Loppacher, M. Bammerlin, E. Meyer, and H. J. Güntherodt, Phys. Rev. Lett. 84, 1172 (2000).

[10] E. Riedo, E. Gnecco, R. Bennewitz, E. Meyer, and H. Brune, Phys. Rev. Lett. 91, 084502 (2003).

[11] A. Socoliuc, R. Bennewitz, E. Gnecco, and E. Meyer, Phys. Rev. Lett. 92, 134301 (2004).

[12] I. Szlufarska, M. Chandross, and R. W. Carpick, J. Phys. D 41, 123001 (2008).

[13] L. Jansen, H. Hölscher, H. Fuchs, and A. Schirmeisen, Phys. Rev. Lett. 104, 256101 (2010). 
[14] T. D. Jacobs and R. W. Carpick, Nat. Nanotechnol. 8, 108 (2013).

[15] N. N. Gosvami, J. A. Bares, F. Mangolini, A. R. Konicek, D. G. Yablon, and R. W. Carpick, Science 348, 102 (2015).

[16] I. Barel, M. Urbakh, L. Jansen, and A. Schirmeisen, Phys. Rev. Lett. 104, 066104 (2010).

[17] Q. Li, T. E. Tullis, D. Goldsby, and R. W. Carpick, Nature (London) 480, 233 (2011).

[18] Y. Liu and I. Szlufarska, Phys. Rev. Lett. 109, 186102 (2012).

[19] K. Tian, N. N. Gosvami, D. L. Goldsby, Y. Liu, I. Szlufarska, and R.W. Carpick, Phys. Rev. Lett. 118, 076103 (2017).

[20] Z. Li and I. Szlufarska, Phys. Rev. Mater. 2 (2018).

[21] Y. Sang, M. Dubé, and M. Grant, Phys. Rev. Lett. 87, 174301 (2001).

[22] A. Vanossi, N. Manini, M. Urbakh, S. Zapperi, and E. Tosatti, Rev. Mod. Phys. 85, 529 (2013).

[23] I. Barel, M. Urbakh, L. Jansen, and A. Schirmeisen, Tribol. Lett. 39, 311 (2010).

[24] I. Barel, M. Urbakh, L. Jansen, and A. Schirmeisen, Phys. Rev. B 84, 115417 (2011).

[25] H. Spikes, Friction 6, 1 (2018).

[26] Y. Shao, T. D. B. Jacobs, Y. Jiang, K. T. Turner, R. W. Carpick, and M. L. Falk, ACS Appl. Mater. Interfaces 9, 35333 (2017).

[27] B. Gotsmann and M. A. Lantz, Phys. Rev. Lett. 101, 125501 (2008).

[28] A. E. Filippov, J. Klafter, and M. Urbakh, Phys. Rev. Lett. 92, 135503 (2004).

[29] See Supplemental Material at http://link.aps.org/ supplemental/10.1103/PhysRevLett.123.116102 for details of experimental setup, sample characterization, friction and adhesion measurements, derivations of equations, and other discussions, which includes Refs. [30-44].

[30] J. L. Hutter and J. Bechhoefer, Rev. Sci. Instrum. 64, 1868 (1993).

[31] M. Varenberg, I. Etsion, and G. Halperin, Rev. Sci. Instrum. 74, 3362 (2003).

[32] H.-B. Liu, N. V. Venkataraman, T. E. Bauert, M. Textor, and S.-J. Xiao, J. Phys. Chem. A 112, 12372 (2008).

[33] N. Sheth, D. Ngo, J. Banerjee, Y. Zhou, C. G. Pantano, and S. H. Kim, J. Phys. Chem. C 122, 17792 (2018).

[34] C. Tripp and M. Hair, Langmuir 11, 1215 (1995).

[35] A. Abramov, A. Keshavarz, and S. Iglauer, J. Phys. Chem. C 123, 9027 (2019).

[36] L. T. Zhuravlev, Colloids Surf. A 173, 1 (2000).

[37] J.-P. Gallas, J.-M. Goupil, A. Vimont, J.-C. Lavalley, B. Gil, J.-P. Gilson, and O. Miserque, Langmuir 25, 5825 (2009).

[38] Y. Yamamoto, K. Sato, K. Nakajima, and K. Kimura, J. Non-Cryst. Solids 499, 408 (2018).

[39] N. N. Gosvami, P. Egberts, and R. Bennewitz, J. Phys. Chem. A 115, 6942 (2011).

[40] L. M. Grant and F. Tiberg, Biophys. J. 82, 1373 (2002).

[41] M. O'Keeffe and P. F. McMillan, J. Phys. Chem. 90, 541 (1986).

[42] M. D. Newton, M. O’Keeffe, and G. V. Gibbs, Phys. Chem. Miner. 6, 305 (1980).

[43] R. Pawlak, W. Ouyang, A. E. Filippov et al., ACS Nano 10, 713 (2016).
[44] A. K. Rappé, C. J. Casewit, K. Colwell, W. A. Goddard, and W. Skiff, J. Am. Chem. Soc. 114, 10024 (1992).

[45] K. Sakai, K. Okada, A. Uka, T. Misono, T. Endo, S. Sasaki, M. Abe, and H. Sakai, Langmuir 31, 6085 (2015).

[46] A. Arcifa, A. Rossi, S. N. Ramakrishna, R. EspinosaMarzal, A. Sheehan, and N. D. Spencer, J. Phys. Chem. C 122, 7331 (2018).

[47] U.D. Schwarz, O. Zwörner, P. Köster, and R. Wiesendanger, Phys. Rev. B 56, 6987 (1997).

[48] U. D. Schwarz, O. Zwörner, P. Köster, and R. Wiesendanger, Phys. Rev. B 56, 6997 (1997).

[49] M. Enachescu, R. J. A. van den Oetelaar, R. W. Carpick, D. F. Ogletree, C. F. J. Flipse, and M. Salmeron, Tribol. Lett., 7, 73 (1999).

[50] M. Enachescu, R. J. A. van den Oetelaar, R. W. Carpick, D. F. Ogletree, C. F. J. Flipse, and M. Salmeron, Phys. Rev. Lett. 81, 1877 (1998).

[51] J. Chen, I. Ratera, J. Y. Park, and M. Salmeron, Phys. Rev. Lett. 96, 236102 (2006).

[52] S. N. Zhurkov, Int. J. Fract. 26, 295 (1984).

[53] E. Evans, Annu. Rev. Biophys. Biomol. Struct. 30, 105 (2001).

[54] O. Dudko, A. Filippov, J. Klafter, and M. Urbakh, Proc. Natl. Acad. Sci. U.S.A. 100, 11378 (2003).

[55] H. Spikes and W. Tysoe, Tribol. Lett. 59, 21 (2015).

[56] O. K. Dudko, G. Hummer, and A. Szabo, Phys. Rev. Lett. 96, 108101 (2006).

[57] G. Hummer and A. Szabo, Biophys. J. 85, 5 (2003).

[58] M. Srinivasan and S. Walcott, Phys. Rev. E 80, 046124 (2009).

[59] P. Sens, Europhys. Lett. 104, 38003 (2013).

[60] J. Blass, M. Albrecht, B. L. Bozna, G. Wenz, and R. Bennewitz, Nanoscale 7, 7674 (2015).

[61] R. Guerra, A. Benassi, A. Vanossi, M. Ma, and M. Urbakh, Phys. Chem. Chem. Phys. 18, 9248 (2016).

[62] J. Blass, M. Albrecht, G. Wenz, R. Guerra, M. Urbakh, and R. Bennewitz, J. Phys. Chem. C 121, 15888 (2017).

[63] Z. Li, L. Pastewka, and I. Szlufarska, Phys. Rev. E 98, 023001 (2018).

[64] K. L. Johnson, Contact Mechanics (Cambridge University Press, Cambridge, England, 1987).

[65] Olver and W. J. Frank, NIST Handbook of Mathematical Functions Hardback and CD-ROM (Cambridge University Press, Cambridge, England, 2010).

[66] J. M. Rimsza, J. Yeon, A. C. T. van Duin, and J. Du, J. Phys. Chem. C 120, 24803 (2016).

[67] S. Perkin, T. Albrecht, and J. Klein, Phys. Chem. Chem. Phys. 12, 1243 (2010).

[68] A. M. Smith, K. R. J. Lovelock, N. N. Gosvami, T. Welton, and S. Perkin, Phys. Chem. Chem. Phys. 15, 15317 (2013).

[69] R. M. Espinosa-Marzal, A. Arcifa, A. Rossi, and N. D. Spencer, J. Phys. Chem. Lett. 5, 179 (2014).

[70] L. A. Jurado, H. Kim, A. Arcifa, A. Rossi, C. Leal, N. D. Spencer, and R. M. Espinosa-Marzal, Phys. Chem. Chem. Phys. 17, 13613 (2015).

[71] H. Li, M. W. Rutland, and R. Atkin, Phys. Chem. Chem. Phys. 15, 14616 (2013).

[72] P. K. Cooper, C. J. Wear, H. Li, and R. Atkin, ACS Sustainable Chem. Eng. 5, 11737 (2017).

[73] M. Han and R. Espinosa-Marzal, Lubricants 6, 64 (2018). 